\title{
The CHESS spectral survey of star forming regions: Peering into the protostellar shock L1157-B1 ${ }^{\star}$
}

\section{Shock dynamics}

B. Lefloch ${ }^{1}$, S. Cabrit ${ }^{2}$, C. Codella ${ }^{3}$, G. Melnick ${ }^{4}$, J. Cernicharo ${ }^{5}$, E. Caux ${ }^{6}$, M. Benedettini ${ }^{7}$, A. Boogert ${ }^{8}$, P. Caselli ${ }^{9}$, C. Ceccarelli ${ }^{1}$, F. Gueth ${ }^{10}$, P. Hily-Blant ${ }^{1}$, A. Lorenzani ${ }^{3}$, D. Neufeld ${ }^{11}$, B. Nisini ${ }^{12}$, S. Pacheco ${ }^{1}$, L. Pagani ${ }^{2}$, J. R. Pardo ${ }^{5}$, B. Parise ${ }^{13}$, M. Salez ${ }^{2}$, K. Schuster ${ }^{10}$, S. Viti ${ }^{12,14}$, A. Bacmann ${ }^{1,15}$, A. Baudry ${ }^{15}$, T. Bell ${ }^{16}$, E. A. Bergin ${ }^{17}$ G. Blake ${ }^{16}$, S. Bottinelli ${ }^{6}$, A. Castets ${ }^{1}$, C. Comito ${ }^{13}$, A. Coutens ${ }^{6}$, N. Crimier ${ }^{1,5}$, C. Dominik ${ }^{18,19}$, K. Demyk ${ }^{6}$, P. Encrenaz ${ }^{2}$, E. Falgarone ${ }^{2}$, A. Fuente ${ }^{20}$, M. Gerin ${ }^{2}$, P. Goldsmith ${ }^{21}$, F. Helmich ${ }^{22}$, P. Hennebelle ${ }^{2}$, T. Henning ${ }^{23}$, E. Herbst ${ }^{24}$, T. Jacq ${ }^{15}$, C. Kahane ${ }^{1}$, M. Kama ${ }^{18}$, A. Klotz ${ }^{6}$, W. Langer ${ }^{21}$, D. Lis ${ }^{16}$, S. Lord ${ }^{16}$, S. Maret ${ }^{1}$, J. Pearson ${ }^{21}$, T. Phillips ${ }^{16}$, P. Saraceno ${ }^{7}$, P. Schilke ${ }^{13,25}$, X. Tielens ${ }^{26}$, F. van der Tak ${ }^{19}$, M. van der Wiel ${ }^{19}$, C. Vastel ${ }^{6}$, V. Wakelam ${ }^{15}$, A. Walters ${ }^{6}$, F. Wyrowski ${ }^{13}$, H. Yorke $^{21}$, R. Bachiller ${ }^{20}$, C. Borys ${ }^{16}$, G. De Lange ${ }^{22}$, Y. Delorme ${ }^{5}$, C. Kramer ${ }^{25,27}$, B. Larsson ${ }^{28}$, R. Lai ${ }^{28}$, F. W. Maiwald ${ }^{21}$, J. Martin-Pintado ${ }^{5}$, I. Mehdi' ${ }^{21}$, V. Ossenkopf ${ }^{25}$, P. Siegel ${ }^{21}$, J. Stutzki ${ }^{24}$, and J. H. Wunsch ${ }^{13}$

(Affiliations are available in the online edition)

Received 31 March 2010 / Accepted 30 April 2010

\section{ABSTRACT}

Context. The outflow driven by the low-mass class 0 protostar L1157 is the prototype of the so-called chemically active outflows. The bright bowshock B1 in the southern outflow lobe is a privileged testbed of magneto-hydrodynamical (MHD) shock models, for which dynamical and chemical processes are strongly interdependent.

Aims. We present the first results of the unbiased spectral survey of the L1157-B1 bowshock, obtained in the framework of the key program "Chemical HErschel Surveys of star forming regions" (CHESS). The main aim is to trace the warm and chemically enriched gas and to infer the excitation conditions in the shock region.

Methods. The CO 5-4 and o- $\mathrm{H}_{2} \mathrm{O} 1_{10}-1_{01}$ lines have been detected at high-spectral resolution in the unbiased spectral survey of the HIFI-band $1 \mathrm{~b}$ spectral window $(555-636 \mathrm{GHz}$ ), presented by Codella et al. in this volume. Complementary ground-based observations in the submm window help establish the origin of the emission detected in the main-beam of HIFI and the physical conditions in the shock.

Results. Both lines exhibit broad wings, which extend to velocities much higher than reported up to now. We find that the molecular emission arises from two regions with distinct physical conditions : an extended, warm $(100 \mathrm{~K})$, dense $\left(3 \times 10^{5} \mathrm{~cm}^{-3}\right)$ component at low-velocity, which dominates the water line flux in Band 1; a secondary component in a small region of B1 (a few arcsec) associated with high-velocity, hot ( $>400 \mathrm{~K}$ ) gas of moderate density $\left((1.0-3.0) \times 10^{4} \mathrm{~cm}^{-3}\right)$, which appears to dominate the flux of the water line at $179 \mu \mathrm{m}$ observed with PACS. The water abundance is enhanced by two orders of magnitude between the low- and the high-velocity component, from $8 \times 10^{-7}$ up to $8 \times 10^{-5}$. The properties of the high-velocity component agree well with the predictions of steady-state C-shock models.

Key words. stars: formation - ISM: jets and outflows - ISM: individual objects: L1157

\section{Introduction}

Shocks in protostellar outflows play a crucial role in the molecular cloud evolution and star formation by transferring momentum and energy back to the ambient medium. There is mounting evidence that these shocks often involve a magnetic precursor where ionic and neutral species are kinematically decoupled. Magneto-hydrodynamical (MHD) shocks are important not only for the cloud dynamics, but also for the chemical evolution through temperature and density changes, which favors the activation of endothermic reactions, ionization, and dust destruction through sputtering and shattering in the ion neutral drift zone. These various processes lead to abundance

* Herschel is an ESA space observatory with science instruments provided by European-led Principal Investigator consortia and with important participation from NASA. enhancements up to several orders of magnitude, as reported for various molecular species in "chemically active" outflows (Bachiller et al. 2001). Conversely, the magnetic field and the ionization fraction play an important role in controlling the size and the temperature of the ion-neutral drift zone. Because of the interplay between the dynamics and chemistry, the physics of MHD shocks requires a comprehensive picture of both the gas and dust physical conditions in the compressed region itself.

Along with $\mathrm{H}_{2}, \mathrm{H}_{2} \mathrm{O}$ and $\mathrm{CO}$ are two key-molecules predicted to dominate the cooling of MHD shocks (Kaufman \& Neufeld 1996). The abundance of $\mathrm{H}_{2} \mathrm{O}$ in protostellar regions can be greatly enhanced in shocks, even of moderate velocity. This occurs both from the sputtering of frozen water from grain mantles and through high-temperature sensitive reactions in the gas phase (Elitzur \& de Jong 1978; Elitzur \& Watson 1978; Bergin et al. 1998). Multi-transition observations of these two 
molecules therefore serve as good probes of shock regions with various excitation conditions, and can be used to set stringent constraints on MHD shock models (Flower \& Pineau des Forets, 2001).

The heterodyne instrument HIFI onboard Herschel allows us to study with unprecedented sensitivity the chemical and dynamical evolution of protostellar shocks, at spectral and angular resolutions comparable to the best ground-based single-dish telescopes. This is the main goal of the spectral survey of L1157-B1, carried out in the guaranteed time key-project CHESS.

The source L1157-mm is a low-mass Class 0 protostar located at a distance estimated between 250 pc (Looney et al. 2007) and 440 pc (Viotti 1969). It drives a spectacular bipolar outflow, which has been studied in detail at millimeter and farinfrared wavelengths. Mapping of the southern lobe of L1157 with the Plateau de Bure Interferometer (PdBI) reveals two limbbrightened cavities (Gueth et al. 1996), each one terminated by a strong bow shock, dubbed "B1" and "B2" respectively (Fig. 1), which are likely the result of episodic ejection events in a precessing, highly collimated jet. The spatial and kinematical structure of B1 has been modelled in great detail by various authors, making it the archetype of protostellar bowshocks in low-mass star-forming regions and the testbed of MHD shock models (Gusdorf et al. 2008a,b).

Here, we report on the emission lines of $\mathrm{CO}$ and $\mathrm{H}_{2} \mathrm{O}$ detected in the low-frequency band of HIFI in the course of the CHESS spectral survey. From comparison with complementary observations, we discuss the origin of the emission, and based on a simple modelling of the source, we derive the water abundance in the shock region.

\section{Observations and results}

A full coverage of the band $1 \mathrm{~b}$ at $20^{\mathrm{h}} 39^{\mathrm{m}} 10.2^{\mathrm{s}}+68^{\circ} 01^{\prime} 10.5^{\prime \prime}$ (J2000) in the bowshock B1 was carried out with the HIFI heterodyne instrument (de Graauw et al. 2010) on board of the Herschel Space Observatory (Pilbratt et al. 2010) during the performance verification phase on 2009 August 1. The corresponding dataset is OBS_1342181160. The HIFI band 1b (from 555.4 to $636.2 \mathrm{GHz}$ ) was covered in double beam switching. Both polarizations ( $\mathrm{H}$ and $\mathrm{V}$ ) were observed simultaneously. The receiver was tuned in double sideband (DSB) with a total integration time of $140 \mathrm{~min}$. In order to obtain the best possible data reconstruction, the survey was acquired with a degree of redundancy of 4 . The wide band spectrometer (WBS) was used as spectrometer, providing a frequency resolution of $0.5 \mathrm{MHz}$.

The data were processed with the ESA-supported package HIPE (Ott et al. 2009). Fits file from level 2 were then created and transformed into GILDAS format ${ }^{1}$ for baseline subtraction and subsequent sideband deconvolution. The spectral resolution was degraded to $1 \mathrm{MHz}$ in the final single sideband (SSB) dataset. The calibration for each receiver ( $\mathrm{H}$ and $\mathrm{V}$ ) is better than $2-3 \%$. The relative calibration between both receivers is also rather good, with a difference in intensity of about $4 \%$. The overall calibration uncertainty is about $7 \%$, except for the strong $\mathrm{CO}$ line present in the band (see below).

Two strong lines dominate over the molecular transitions detected in the spectral band: the fundamental line of water in its ortho state $\mathrm{o}-\mathrm{H}_{2} \mathrm{O}_{10}-1_{01}$ at $556.936069 \mathrm{GHz}$ and the $\mathrm{CO}$ 5-4 line at $576.276905 \mathrm{GHz}$ (Fig. 1). The final rms noise is $13 \mathrm{mK}$. We adopted the theoretical telescope main-beam efficiency $\eta_{\mathrm{mb}}=0.723$, and a main-beam size of $39^{\prime \prime}(\mathrm{HPFW})$ in

${ }^{1}$ http://wWw.iram.fr/IRAMFR/GILDAS
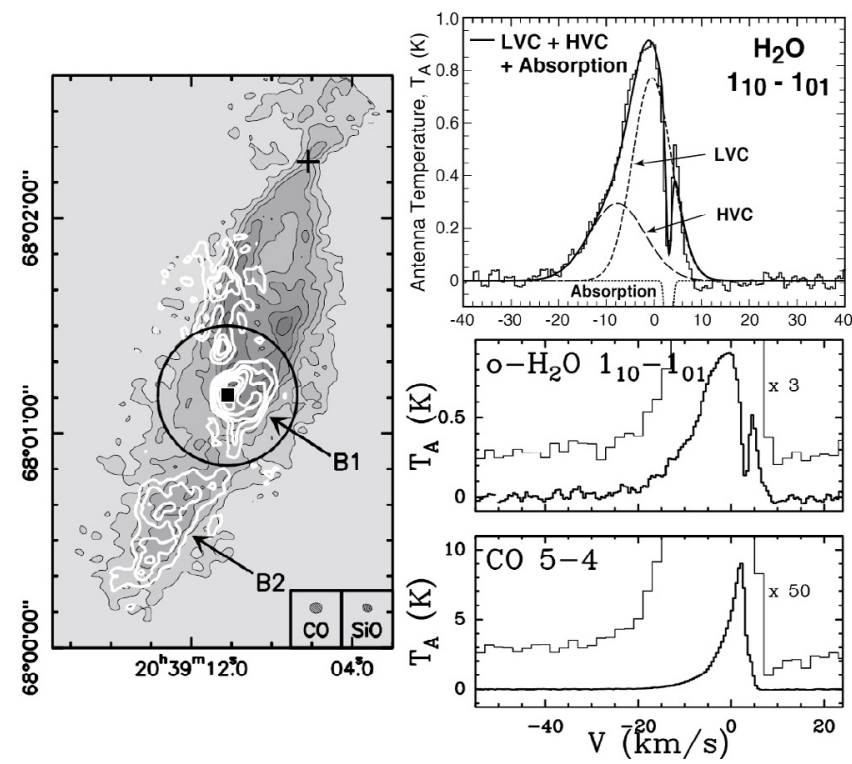

Fig. 1. (left) Southern outflow lobe of L1157 in CO 1-0 (greyscale and black contours) and in $\mathrm{SiO} 2-1$ (white contours) as observed at the PdBI (Gueth et al. 1996, 1998). A black square marks the nominal position of bowshock L1157-B1 observed with HIFI. The HIFI main-beam is represented with a black circle. (right) Panel of the CO 5-4 (bottom) and $\mathrm{o}-\mathrm{H}_{2} \mathrm{O} 1_{10}-1_{01}$ (centre) line spectra obtained in band $1 \mathrm{~b}$ of HIFI. For both lines, we show (dashed) a magnified and spectrally smoothed view of the emission. Intensities are expressed in units of antenna temperature.(top) $\mathrm{H}_{2} \mathrm{O}$ spectrum with fitted low-velocity component (LVC), high-velocity component (HVC), absorption feature and summed fitted spectrum.

the whole band. Unless indicated, intensities are expressed in units of antenna temperature $T_{\mathrm{A}}$.

The CO 5-4 transition is detected with an intensity of $9 \mathrm{~K}$ $\left(T_{\mathrm{A}}\right)$ and a linewidth of $5 \mathrm{~km} \mathrm{~s}^{-1}$. We notice a weak absorption feature in the line profile in the redshifted gas over a wide velocity range, which may partly arise from cloud contamination in the reference position. The intensity in the blue wing of the CO line differs by as much as $20 \%$ between both polarizations. This effect is not observed towards the $\mathrm{H}_{2} \mathrm{O}$ line. Its origin is not understood at the moment.

The fundamental o- $\mathrm{H}_{2} \mathrm{O} 1_{10}-1_{01}$ line is detected with an intensity of $0.9 \mathrm{~K}\left(T_{\mathrm{A}}\right)$ at the peak. It is characterized by a broad linewidth $\approx 15 \mathrm{~km} \mathrm{~s}^{-1}$. The line displays an absorption dip at $v_{\mathrm{lsr}}=+2.9 \mathrm{~km} \mathrm{~s}^{-1}$ and a broad redshifted wing extending up to $+8 \mathrm{~km} \mathrm{~s}^{-1}$. The broad linewidth of the $\mathrm{H}_{2} \mathrm{O}$ spectrum could be fit with three Gaussian velocity components, a low-velocity, a high-velocity, and an absorption component. The low (high) velocity component peaks at $v_{\mathrm{lsr}}=-0.58 \mathrm{~km} \mathrm{~s}^{-1}$ $\left(-7.86 \mathrm{~km} \mathrm{~s}^{-1}\right)$; the linewidth and peak intensity derived from the fit are $9.56 \mathrm{~km} \mathrm{~s}^{-1}$ and $0.77 \mathrm{~K}\left(13.72 \mathrm{~km} \mathrm{~s}^{-1}\right.$ and $\left.0.29 \mathrm{~K}\right)$, respectively. The absorption component was fit by a narrow line Gaussian $\left(\Delta V=1.38 \mathrm{~km} \mathrm{~s}^{-1}\right)$ of amplitude $-0.48 \mathrm{~K}$ centered at $v_{\text {lsr }}=+2.9 \mathrm{~km} \mathrm{~s}^{-1}$. The fit of the individual components and the resulting fit to the water spectrum is displayed in Fig. 1.

Overall, the $\mathrm{H}_{2} \mathrm{O}$ and $\mathrm{CO}$ emission are detected in the same velocity range. The high sensitivity of the HIFI observations permits the detection of emission from the entrained gas up to $v_{\text {lsr }}=-30 \mathrm{~km} \mathrm{~s}^{-1}$, i.e. about $10 \mathrm{~km} \mathrm{~s}^{-1}$ higher than was previously known from ground based observations. However, line profiles differ noticeably and the ratio of the $\mathrm{H}_{2} \mathrm{O} / \mathrm{CO} 5-4$ line intensities increases with increasing velocities from about 0.2 in the ambient gas up to 0.9 at $\mathrm{v}_{\mathrm{lsr}}=-25 \mathrm{~km} \mathrm{~s}^{-1}$ (Fig. 3). 


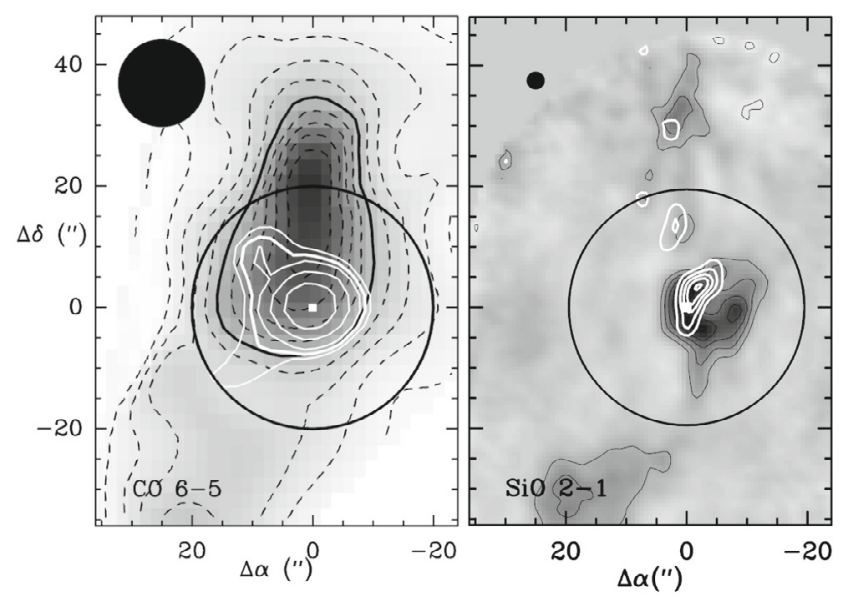

Fig. 2. (left) Velocity-integrated CO 6-5 emission maps of the lowand high-velocity components. LVC (HVC) emission is represented in greyscale and thin dashed contours (white contours); first contour and contour interval are $3 \sigma$ and $1 \sigma$ ( $10 \%$ of the peak flux), respectively, Contours at half-power are drawn in thick. (right) Same for SiO 2-1 observed at the PdbI. The HIFI main-beam is represented with a black circle.

All the other molecular tracers detected in the HIFI band show a pronounced break in the line profile at $v_{\mathrm{lsr}} \approx-7.2 \mathrm{~km} \mathrm{~s}^{-1}$ (Codella et al. this volume). This is also observed in the $\mathrm{CO}$ 65 spectrum of $\mathrm{B} 1$ obtained by us at the CSO, as part of complementary observations to help analyse the CHESS data. The maps of the whole southern lobe of L1157 in CO 3-2 and 6-5 obtained at the CSO in June 2009, with 24" and 14.5" respectively, will be discussed in detail in a forthcoming paper (Lefloch et al. 2010, in prep.).

Below, we define the region with $v_{\mathrm{lsr}}<-7.25 \mathrm{~km} \mathrm{~s}^{-1}$ as the high-velocity component, hereafter HVC (see also Codella et al), and the region with $v_{\mathrm{lsr}}>-7.25 \mathrm{~km} \mathrm{~s}^{-1}$ ) as the low-velocity component (LVC). As we discuss below, these two velocity components are characterized by different spatial extents and excitation conditions.

\section{Discussion}

\subsection{Origin of the emission}

Due to its relatively high energy above the ground state $\left(E_{\text {up }}=\right.$ $116 \mathrm{~K}$ ) the $\mathrm{CO} 6-5$ transition is a good probe of the warm regions where $\mathrm{H}_{2} \mathrm{O}$ can evaporate from grain mantles and be released in the gas phase. SiO 2-1, observed at the PdBI at 2.5" resolution is a particularly good tracer of shocks strong enough to release refractory elements in the gas phase, because it is usually undetected in the cold, quiescent molecular gas.

The overall $\mathrm{SiO}$ emission is strongly peaked at the position of $\mathrm{B} 1$, which appears as a region of $\approx 15^{\prime \prime}$ size located at the apex of the cavity. Interferometric maps of the southern lobe (Figs. 1-2) reveal extended emission along the eastern wall of the cavity (the low-velocity wing of the bow) and downstream of $\mathrm{B} 1$, at velocities close to systemic, both blue and red. By comparing these data with IRAM 30m observations (Bachiller et al. 2001), we checked that unlike the HVC, a fraction of the flux emitted in the LVC is actually missed in the PdBI data, which is direct evidence for extended emission. This is consistent with the CO 6-5 data (Fig. 2). The low-velocity gas emission is located in the wake of B1, reaching $\sim 40^{\prime \prime}$ North from the apex. The area of the LVC amounts to $\approx 1 / 3$ of the HIFI beam (see Fig. 2). Interestingly, the PACS map of the $\mathrm{H}_{2} \mathrm{O} 179 \mu \mathrm{m}$ line reveals large-scale emission, spatially coinciding with $\mathrm{SiO} 2-1$ emission in the outflow (Nisini et al. 2010).
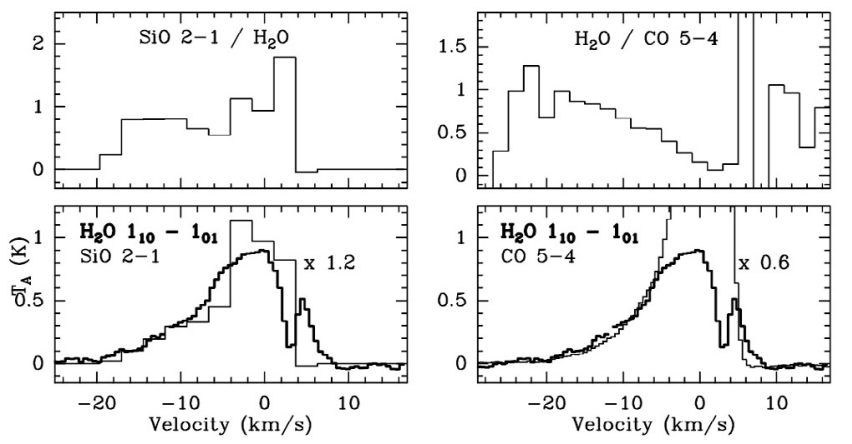

Fig. 3. (bottom left) Comparison of the o- $\mathrm{H}_{2} \mathrm{O} 1_{10}-1_{01}$ line profile with the $\mathrm{SiO} 2-1$ emission observed at the PdBI, averaged over the HIFI beam. (top left) Variations of the $\mathrm{SiO} 2-1 / \mathrm{H}_{2} \mathrm{O}$ line ratio as a function of velocity. (bottom right) Comparison of the o- $\mathrm{H}_{2} \mathrm{O} 1_{10}-1_{01}$ and CO 5-4 line profiles. (top right) Variations of the $\mathrm{H}_{2} \mathrm{O} / \mathrm{CO} 5-4$ line ratio as a function of velocity, smoothed to a resolution of $2 \mathrm{~km} \mathrm{~s}^{-1}$.

In any case, there is definitely much less molecular gas emission associated with the western wall of the cavity (Benedettini et al. 2007). We therefore expect an asymmetry in the $\mathrm{H}_{2} \mathrm{O}$ spatial distribution, similar to that observed in many other tracers such as CS or HCN, as shown by the PACS map of the $179 \mu \mathrm{m}$ $\mathrm{H}_{2} \mathrm{O}$ line (Nisini et al. 2010).

We note an excellent agreement between the $\mathrm{H}_{2} \mathrm{O}$ and the low-excitation $\mathrm{SiO} 2-1$ line profiles (Fig. 3) in the high-velocity range, with a constant $\mathrm{SiO} 2-1 / \mathrm{H}_{2} \mathrm{O}$ line ratio $\approx 0.8$ between -20 and $-7 \mathrm{~km} \mathrm{~s}^{-1}$. This has important implications. First, this constant ratio in the range of the HVC suggests that both emissions most likely arise from the same region and that the emissions are optically thick. In that case, the low intensities measured in the high-velocity component ( $\mathrm{a}$ few tenths of $\mathrm{K}$ ) point to a small size extent. This is direct evidence that the $\mathrm{H}_{2} \mathrm{O}$ emission detected fills only partly the HIFI beam. Indeed, the bulk of the SiO HVC originates from a small region of $4^{\prime \prime} \times 12^{\prime \prime}$ in B1 (Fig. 2), corresponding to a filling factor $f f \sim 0.03$ in the HIFI main-beam. Second, if silicon comes from grain erosion, the $\mathrm{SiO}$ profile is predicted to be much narrower than $\mathrm{H}_{2} \mathrm{O}$ because it takes a long time for $\mathrm{Si}$ to oxidize into $\mathrm{SiO}$, so $\mathrm{SiO}$ comes only from the cold postshock, as discussed by Gusdorf et al. (2008b). The similarity of the $\mathrm{SiO}$ and $\mathrm{H}_{2} \mathrm{O}$ line profiles suggests that $\mathrm{SiO}$ forms more extensively in the shock than predicted by oxidation of sputtered $\mathrm{Si}$ atoms. As it can be released in the gas phase even at low velocities in the shock, $\mathrm{SiO}$ is present in the gas phase over the full width of the shock wave.

In summary, we find strong observational evidence that the emission from the HVC and LVC arises from regions of different physical extent. The size of the HVC appears definitely much lower than the $\operatorname{LVC}$ ( $f f \simeq 0.03$ and 0.3 , respectively). It is true however that the present determinations are uncertain. HIFI observations of the high-excitation lines of $\mathrm{CO}$ and $\mathrm{H}_{2} \mathrm{O}$ will make it possible to better establish appropriate filling factors for these components.

\subsection{Physical conditions}

We first estimated the physical conditions from the emission detected in the CO 3-2, 5-4, and 6-5 transitions both in HVC and LVC. We modelled each velocity component as a simple uniform slab, adopting the size (filling factor) estimated above. Calculations were done in the large-velocity gradient approach, using the $\mathrm{CO}$ collisional coefficients determined by Flower (2001) for ortho- $\mathrm{H}_{2}$ collisions in the range $5 \mathrm{~K}-400 \mathrm{~K}$. For temperatures beyond $400 \mathrm{~K}$, the collisional coefficients were extrapolated adopting a temperature dependence of $\sqrt{T / 400 \mathrm{~K}}$. 
A\&A 518, L113 (2010)

Table 1. Observed and fitted parameters of the $\mathrm{H}_{2} \mathrm{O}$ and CO 5-4 lines and prediction for the $179 \mu \mathrm{m} \mathrm{H}_{2} \mathrm{O}$ line flux.

\begin{tabular}{ccccccccccc}
\hline \hline & $\begin{array}{c}\mathrm{H}_{2} \mathrm{O} 557 \mathrm{GHz} \\
\left(\mathrm{K} \mathrm{km} \mathrm{s}^{-1}\right)\end{array}$ & $\begin{array}{c}\mathrm{CO} \mathrm{5-4} \\
\left(\mathrm{K} \mathrm{km} \mathrm{s}^{-1}\right)\end{array}$ & $\begin{array}{c}\mathrm{CO} \mathrm{6-5} \\
\left(\mathrm{K} \mathrm{km} \mathrm{s}^{-1}\right)\end{array}$ & $\begin{array}{c}\mathrm{CO} \mathrm{3-2} \mathrm{K} \mathrm{km} \mathrm{s}^{-1} \\
\left.\mathrm{Km}^{\prime \prime}\right)\end{array}$ & $\begin{array}{c}\mathrm{Size} \\
\left({ }^{\prime \prime}\right.\end{array}$ & $\begin{array}{c}N(\mathrm{CO})^{2} \\
\left(\mathrm{~cm}^{-2}\right)\end{array}$ & $\begin{array}{c}\mathrm{n}\left(\mathrm{H}_{2}\right)^{2} \\
\left(\mathrm{~cm}^{-3}\right)\end{array}$ & $\begin{array}{c}T^{2} \\
(\mathrm{~K})\end{array}$ & $\begin{array}{c}X\left(\mathrm{H}_{2} \mathrm{O}\right)^{3} \\
\left(\mathrm{~cm}^{-2}\right)\end{array}$ & $\begin{array}{c}F(179 \mu \mathrm{m}) \\
\left(\mathrm{W} \mathrm{cm}^{-2}\right)\end{array}$ \\
\hline LVC & 7.83 & 45.4 & 29.2 & 40.4 & 25 & $8.0(16)$ & $(1.0-3.0)(5)$ & 100 & $0.8(-6)$ & $4.2(-20)$ \\
HVC & 4.28 & 3.98 & 1.34 & 3.11 & 7 & $5.0(16)$ & $(1.0-3.0)(4)$ & 400 & $0.8(-4)$ & $7.1(-20)$ \\
\hline
\end{tabular}

Notes. The CO fluxes in the HVC and LVC are integrated in the velocity intervals $[-30 ;-7.25]$ and $[-7.25 ;+11.0]$, respectively. The $\mathrm{H}_{2} \mathrm{O}$ fluxes are derived from a multiple Gaussian fit to the line profile. Intensities are expressed in units of antenna temperature $\left(T_{\mathrm{A}}\right) \mathrm{K} \mathrm{km} \mathrm{s}^{-1}$. ${ }^{(1)} \mathrm{From} \mathrm{CSO}$ observations smoothed down to the resolution of the HIFI observations; ${ }^{(2)}$ determined from LVG analysis of the CO emission; ${ }^{(3)}$ from comparison of LVG-derived $\mathrm{N}\left(\mathrm{o}-\mathrm{H}_{2} \mathrm{O}\right)$ with $\mathrm{N}(\mathrm{CO})$, assuming a water OPR of 3 and an abundance $[\mathrm{CO}] /\left[\mathrm{H}_{2}\right]=10^{-4}$.
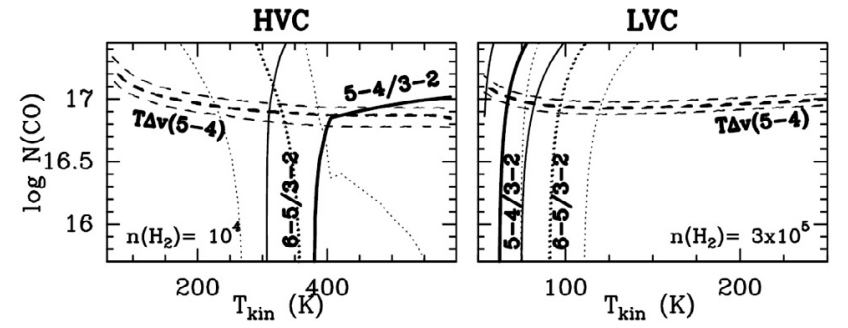

Fig. 4. Best-fit solution to the LVG modelling of CO line temperatures in the 3-2, 5-4 and 6-5 transitions for both HVC (left) and LVC (right) components, respectively. The contour of the observed CO 5-4 integrated line area $(T \Delta v)$, corrected for main-beam dilution, is drawn in a dashed line, as well as the 5-4/3-2 and 6-5/3-2 line ratios (solid and dotted lines, respectively). Thin lines delineate the uncertainties in the observed values.

Both components appear to have the same gas column density $\mathrm{N}(\mathrm{CO}) \simeq 10^{17} \mathrm{~cm}^{-2}$ (Fig. 4). We found the high-velocity gas component to be unambiguously associated with hot gas $(T>350 \mathrm{~K})$ of moderate density $\approx 3.0 \times 10^{4} \mathrm{~cm}^{-3}$, whereas the low-velocity component arises from gas at a lower temperature $(100 \mathrm{~K})$ and higher density $\left(\sim 3.0 \times 10^{5} \mathrm{~cm}^{-3}\right)$. The temperature estimated for the extended component agree reasonably well with other determinations from $\mathrm{NH}_{3}$ and $\mathrm{CH}_{3} \mathrm{CN}$ (Tafalla \& Bachiller 1995; Codella et al. 2009).

With the physical conditions derived from the CO analysis, we modelled the integrated intensity and the line profile of the $\mathrm{o}-\mathrm{H}_{2} \mathrm{O} 1_{10}-1_{01}$ transition as well as the reported PACSmeasured $179 \mu \mathrm{m} \mathrm{H}_{2} \mathrm{O}$ line intensity $\left(\sim 10^{-19} \mathrm{~W} \mathrm{~cm}^{-2}\right.$, Nisini et al. 2010) to compute the total ortho water abundance in each velocity component. We used a radiative transfer code in the large-velocity gradient approach (and slab geometry) detailed in Melnick et al. (2008), taking into account an ortho to para ratio (OPR) of 1.2 for $\mathrm{H}_{2}$, as derived from Spitzer (Neufeld et al. 2009). Here, we assume the absorption component at $+2.9 \mathrm{~km}$ is due to foreground gas unrelated to L1157-B1. Together, the two components of the o- $\mathrm{H}_{2} \mathrm{O} 1_{10}-1_{01}$ line produce a total $\mathrm{H}_{2} \mathrm{O}$ $212-1_{01} 179 \mu \mathrm{m}$ line flux of $1.1 \times 10^{-19} \mathrm{~W} \mathrm{~cm}^{-2}$. For the temperature range derived from our $\mathrm{CO}$ analysis, we estimated ortho- $\mathrm{H}_{2} \mathrm{O}$ column densities of $(4.0-5.0) \times 10^{14} \mathrm{~cm}^{-2}$ and $(2.5-3.0) \times 10^{16} \mathrm{~cm}^{-2}$ for the LVC and the HVC, respectively. Assuming an OPR of 3 , we derived the $\mathrm{H}_{2} \mathrm{O}$ abundance from comparison with the gas column densities estimated from $\mathrm{CO}$ (see Table 1). We obtained an abundance ratio $\left[\mathrm{H}_{2} \mathrm{O}\right] /[\mathrm{CO}] \simeq$ 0.8 in the high-velocity gas, which is consistent with previous results from ODIN (Benedettini et al. 2002) and agrees reasonably well with the predictions of steady-state C-shock models for this set of physical parameters (shock velocity $V_{\mathrm{s}} \simeq 20 \mathrm{~km} \mathrm{~s}^{-1}$, pre-shock density $n\left(\mathrm{H}_{2}\right)=5 \times 10^{3} \mathrm{~cm}^{-3}$; Gusdorf et al. 2008a,b).

An interesting prediction of our model is that the HVC contribution to the $179 \mu$ m flux dominates over the LVC contribution (see last column in Table 1). The higher temperature of this component drives the neutral-neutral reactions that efficiently form $\mathrm{H}_{2} \mathrm{O}$, and the higher shock velocity can more efficiently remove water from grain mantles (see Melnick et al. 2008), resulting in the much greater ortho- $\mathrm{H}_{2} \mathrm{O}$ column density than in the LVC. Comparison with $\mathrm{NH}_{3}$ also suggests that the water production in the HVC is strongly dominated by high-temperature reactions (see Codella et al.). The higher ortho- $\mathrm{H}_{2} \mathrm{O}$ column density is what produces the higher $179 \mu \mathrm{m}$ line flux from this component. Consistent results are obtained by Nisini et al. (2010) based on a $179 \mu \mathrm{m}$ PACS map and previous ODIN and SWAS observations of the $\mathrm{o}-\mathrm{H}_{2} \mathrm{O} 1_{10}-1_{01}$ line, assuming one single physical component dominates the water line emission in the HIFI beam. Follow-up observations of the higher-excitation lines of $\mathrm{CO}$ and $\mathrm{H}_{2} \mathrm{O}$ with HIFI will help us constrain more accurately the physical conditions of each velocity component (density, temperature) and more generally in the shock.

Acknowledgements. HIFI has been designed and built by a consortium of institutes and university departments from across Europe, Canada and the United States under the leadership of SRON Netherlands Institute for Space Research, Groningen, The Netherlands and with major contributions from Germany, France and the US. Consortium members are: Canada: CSA, U.Waterloo; France: CESR, LAB, LERMA, IRAM; Germany: KOSMA, MPIfR, MPS; Ireland, NUI Maynooth; Italy: ASI, IFSI-INAF, Osservatorio Astrofisico di Arcetri- INAF; Netherlands: SRON, TUD; Poland: CAMK, CBK; Spain: Observatorio Astronómico Nacional (IGN), Centro de Astrobiología (CSIC-INTA). Sweden: Chalmers University of Technology - MC2, RSS \& GARD; Onsala Space Observatory; Swedish National Space Board, Stockholm University - Stockholm Observatory; Switzerland: ETH Zurich, FHNW; USA: Caltech, JPL, NHSC. HIPE is a joint development by the Herschel Science Ground Segment Consortium, consisting of ESA, the NASA Herschel Science Center, and the HIFI, PACS and SPIRE consortia.

\section{References}

Bachiller, R., \& Perez-Gutierrez, M. 1997, ApJ, 487, L93

Bachiller, R., Perez-Gutierrez, M., Kumar, M. S. N., \& Tafalla, M. 2001, A\&A, 372,899

Benedettini, M., Visit, S., Giannini, T., et al. 2002, A\&A, 395, 657

Benedettini, M., Viti, S., Codella, C., et al. 2007, MNRAS, 381, 1127

Bergin, E. A., Neufeld, D. A., \& Melnick, G. J. 1998, ApJ, 499, 777

Codella, C., Benedettini, M., Beltrán, M., et al. 2009, A\&A, 507, L25

Codella, C., et al. 2010, A\&A, 518, L144

de Graauw, Th., et al. 2010, A\&A, 518, L6

Elitzur, M., \& de Jong, T. 1978, A\&A, 67, 323

Elitzur, M., \& Watson, W. D., ApJ, 70, 443

Flower, D. R. 2001, J. Phys. B, 34, 2731

Flower, D. R., \& Pineau des Forets, G. 2010, MNRAS, accepted

Gueth, F., Guilloteau, S., \& Bachiller, R. 1996, A\&A, 307, 891

Gueth, F., Guilloteau, S., \& Bachiller, R. 1998, A\&A, 333, 287

Gusdorf, A., Cabrit, S., Flower, D. R., et al. 2008a, A\&A, 482, 809

Gusdorf, A., Pineau des Forets, G., Cabrit, S., et al. 2008b, A\&A, 490, 695

Kaufman, M., \& Neufeld, D. 1996, ApJ, 456, 611

Looney, L. W., Tobin, J. J., \& Kwon, W. 2007, ApJ, 670, L131

Melnick, G. J., Tolls, V., Neufeld, D. A., et al. 2008, ApJ, 683, 876

Neufeld, D. A., Giannini, T., Melnick, G. J., et al. 2009, ApJ, 706, 170

Nisini, B., et al. 2010, A\&A, 518, L120

Ott, S. 2009, in Astronomical Data Analysis Software and Systems XIX, eds.

Y. Mizumoto, K.-I. Morita, \& M. Ohishi, ASP Conf. Ser., in press

Pilbratt, G. L., et al. 2010, A\&A, A\&A, 518, L1

Viotti, N. R. 1969, Mem. Soc. Astron. Ital., 40, 75 
B. Lefloch et al.: CHESS survey of L1157-B1 : Shock dynamics. II.

1 Laboratoire d'Astrophysique de Grenoble, UMR 5571-CNRS, Université Joseph Fourier, Grenoble, France

e-mail: lefloch@obs.ujf-grenoble.fr

2 Observatoire de Paris-Meudon, LERMA UMR CNRS 8112. Meudon, France

3 INAF, Osservatorio Astrofisico di Arcetri, Firenze, Italy

4 Center for Astrophysics, Cambridge MA, USA

${ }^{5}$ Centro de Astrobiología, CSIC-INTA, Madrid, Spain

${ }^{6}$ CESR, Université Toulouse 3 and CNRS, Toulouse, France

7 INAF, Istituto di Fisica dello Spazio Interplanetario, Roma, Italy

${ }^{8}$ IPAC, NASA Herschel Science Center, CalTech, USA

9 School of Physics and Astronomy, University of Leeds, Leeds, UK

${ }_{10}$ Institut de Radio Astronomie Millimétrique, Grenoble, France

11 Johns Hopkins University, Baltimore MD, USA

12 INAF, Osservatorio Astronomico di Roma, Monte Porzio Catone, Italy

13 Max-Planck-Institut für Radioastronomie, Bonn, Germany

14 Department of Physics and Astronomy, University College London, London, UK

15 Université de Bordeaux, Laboratoire d'Astrophysique de Bordeaux, France; CNRS/INSU, Floirac, France

16 California Institute of Technology, Pasadena, USA

17 University of Michigan, Ann Arbor, USA

18 Astronomical Institute "Anton Pannekoek", University of Amsterdam, Amsterdam, The Netherlands

19 Department of Astrophysics/IMAPP, Radboud University Nijmegen, Nijmegen, The Netherlands

20 IGN Observatorio Astronómico Nacional, Spain

21 Jet Propulsion Laboratory, Caltech, Pasadena, CA 91109, USA

22 SRON, Groningen, The Netherlands

${ }^{23}$ Max Planck Institut für Astronomie, Heidelberg - Germany Ohio State University, Columbus, OH, USA

24 Physikalisches Institut, Universität zu Köln, Köln, Germany

25 Leiden Observatory, Leiden University, Leiden, The Netherlands

${ }^{26}$ IRAM, Granada, Spain

27 Department of Astronomy, Stockholm University, Stockholm, Sweden

28 Northrop Grumman Aerospace Systems, Redondo Beach, CA 90278, USA 